$\underline{\text { Avicenna Journal of Nursing and Midwifery Care - ISSN 2676-5748 }}$

\title{
Impact of Inhalation Aromatherapy with Damask Rose on Anxiety of Patients Undergoing Coronary Angiography: A Single-Blind Randomized Controlled Clinical Trial
}

\author{
Ali Bikmoradi', Ghodratollah Roshanaei², Shirin Moradkhani ${ }^{3}$, Azad Fatahi** \\ 1. PhD in Health Care Management, Associate Professor, Department of Medical Surgical Nursing, School of Nursing \\ and Midwifery, Hamadan University of Medical Sciences, Hamadan, Iran \\ 2. PhD in Biostatistics, Assistant Professor, Department of Biostatistics and Epidemiology, Modeling Non- \\ communicable Diseases Research Center, School of Public Health, Hamadan University of Medical Sciences, \\ Hamadan, Iran \\ 3. PhD in pharmacognosy, Assistant Professor, Department of Pharmacognosy, School of Pharmacy, Hamadan \\ University of Medical Sciences, Hamadan, Iran \\ 4. MSc Student, Student Research Committee, School of Nursing and Midwifery, Hamadan University of Medical \\ Sciences, Hamadan, Iran
}

\section{Article Info}

Received: 2019/07/29;

Accepted: 2019/09/11;

Published Online: 2020/06/24

$\underline{10.30699 / a j n m c .28 .2 .93}$

Original Article

Use your device to scan and read the article online

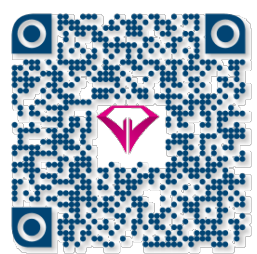

\section{ABSTRACT}

Introduction: Coronary artery angiography is a common diagnostic procedure in cardiovascular diseases which could cause fear, stress, anxiety and probably some instability at hemodynamic parameters in the patients before this diagnostic procedure. This study aimed to assess impact of inhalation aromatherapy with damask rose on anxiety of patients undergoing coronary angiography.

Methods: A single-blinded randomized controlled trial was conducted with 98 patients at aromatherapy and control group undergoing coronary angiography in the Besat educational hospital of Hamadan, Iran, in 2017. Patients inhaled 5 drops of $40 \%$ Rosa Damask essential oil for 20 minutes before the angiography at aromatherapy group and inhaled 5 drops of distilled water for $20 \mathrm{~min}$ before the angiography at the control group. Anxiety was measured before and 2 minutes after intervention by state section of state-trait anxiety inventory (STAI) questionnaire. Data were analyzed by SPSS 16 using independent t-test and paired t-test.

Results: There was no significant difference between mean of patient's anxiety at aromatherapy and control group before intervention. There was significant difference between mean of patient's anxiety before and after intervention at the aromatherapy group $(P<0.001)$ which could decrease mean of patient's anxiety. In contrast, there was no significant difference between mean of patient's anxiety before and after intervention at control group $(P=0.067)$.

Conclusion: Inhalation aromatherapy with Rosa damask essential oil $40 \%$ could have a good impact on decreasing anxiety at patients undergoing coronary angiography. Rosa damask essential oil $40 \%$ can be used as a complementary therapy to reduce anxiety in patients prior to coronary angiography.

Keywords: Complementary therapies, Inhalational aromatherapy, Rosa damask, Anxiety, Coronary angiography

Copyright $($ ) 2020, This is an original open-access article distributed under the terms of the Creative Commons Attribution-noncommercial 4.0 International License which permits

\section{How to Cite This Article:}

Bikmoradi A, Roshanaei G, Moradkhani S, Fatahi A. Impact of inhalation aromatherapy with damask rose on anxiety of patients undergoing coronary angiography: A single-blind randomized controlled clinical trial. Avicenna J Nurs Midwifery care. 2020; 28 (2):93-102

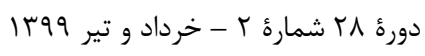

$$
\text { مجله مراقبت يرستارى و مامايى ابنسينا }
$$




\title{
تأثير رايحهدرمانى استنشاقى با اسانس كل رز بر اضطراب بيماران تحت آنزيوكرافى عروق كرونرى يكى كار آزمايى بالينى يك سوكور
}

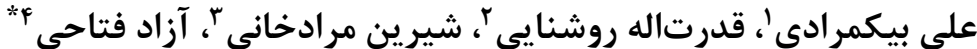

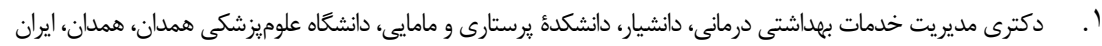

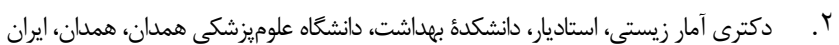

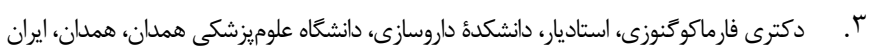

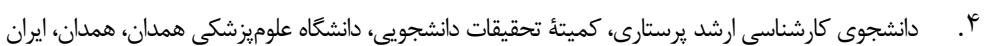

\begin{tabular}{|c|c|}
\hline جكيده & اطلاعات مقاله \\
\hline 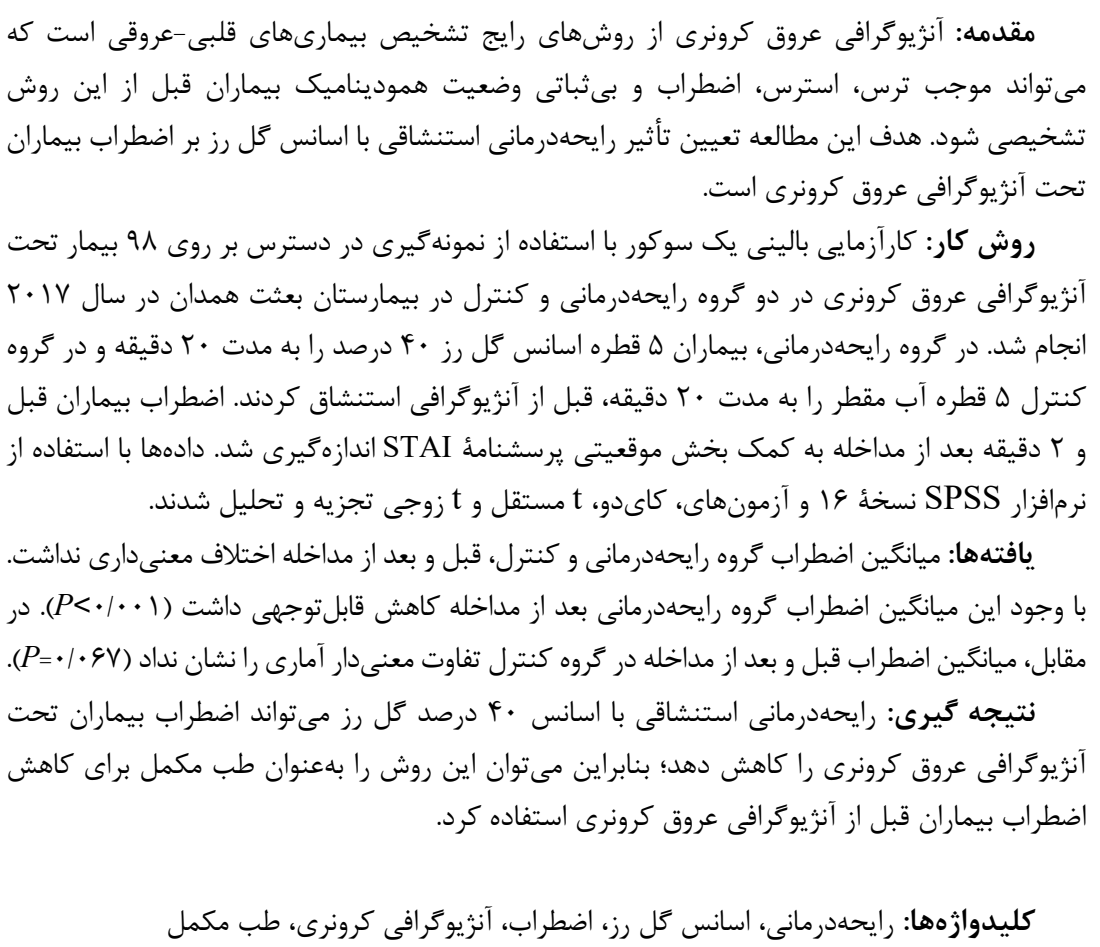 & 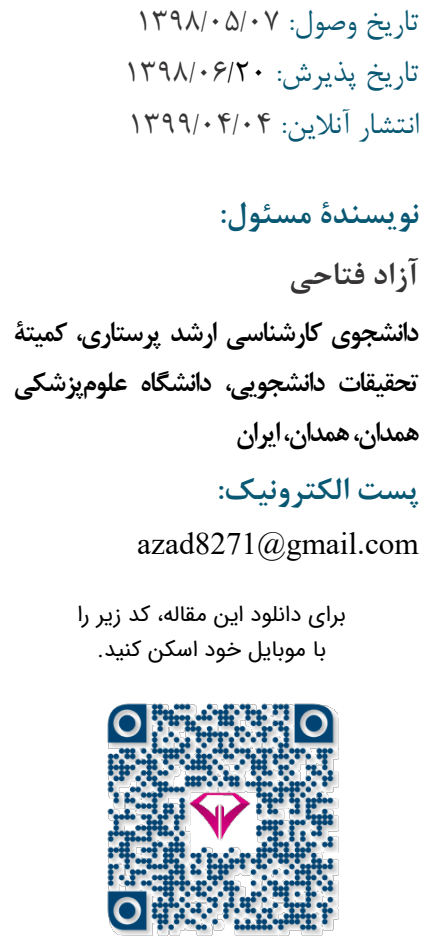 \\
\hline
\end{tabular}

مقدمه

با داشتن حساسيت تشخيصى بالا، براى مدتى طولانى

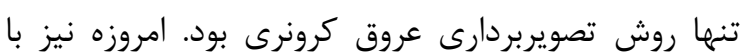
وجود روشهاى غيرتهاجمى، آنزيوگرافى تهاجمى هنوز استاندارد طلايى تشخيص بيمارىهاى عروق كرونرى است است إن

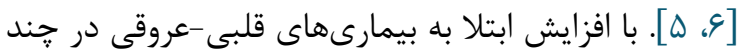
سال اخير، استفاده از روشهاى تشخيصى پيشرفته ازجمله

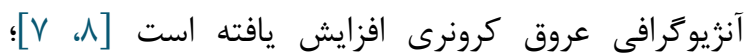

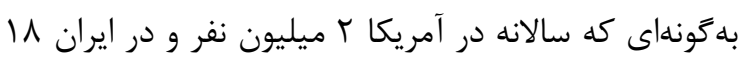
هزار نفر تحت آنزيوگرافى عروق كرونرى قرار مى

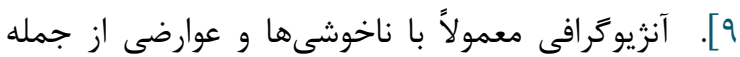

با وجود يِيشرفتهاى اخير در شناخت ياتوفيزيولوزى و عوامل خطر بيمارىهاى قلبى-عروقى، اين بيمارىها هنوز

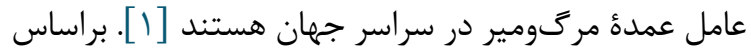

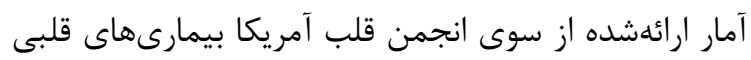

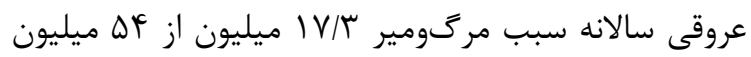

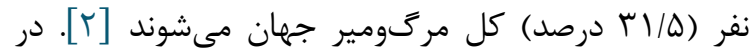
ايران نيز شايعترين عامل مركَمير در افراد بالغ مشاهده

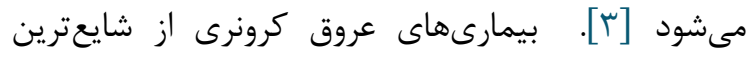
بيمارىهاى قلبى-عروقى است كه بهصورت گستردهاى از

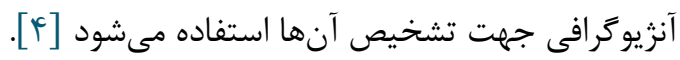


روشبر رسى

مطالعُ حاضر يك كار آزمايى بالينى است كه جامعه

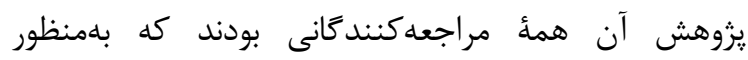

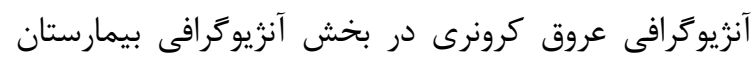

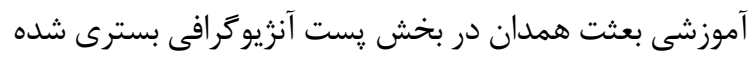

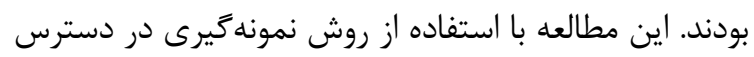
در دو گروه رايحهدرمانى و كنترل بر روى يل9 بيمار انجام شد.

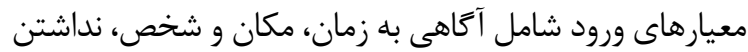

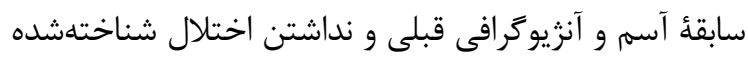

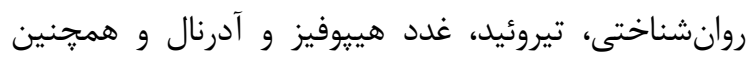
مصرفنكدرن داروهاى روانى بود. معيارهاى خروج از مطالعه

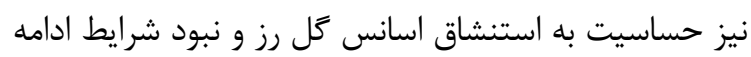
شركت در مطالعه بود. تعداد نمونه با در نظر كرفتن خطاى

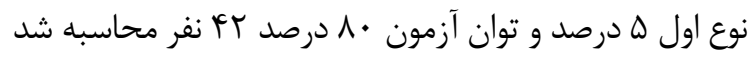

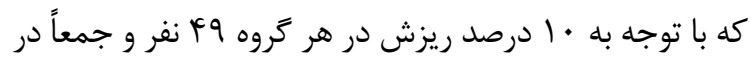

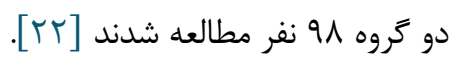

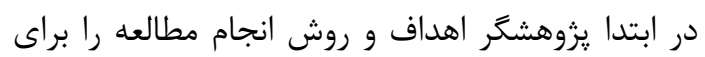
مديران بيمارستان، يزشكان و يرستاران بخش و همرجنين

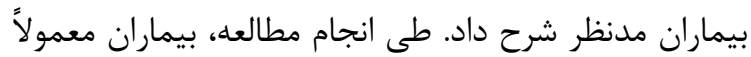

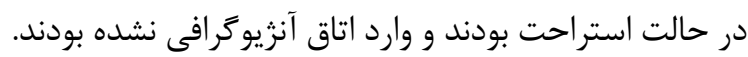
يك ساعت قبل از انجام آنزيوگرافى عروق كرونرى يرسشنامئ جمعيتشناختى و بخش اضطراب موقعيتى يرسشنامة

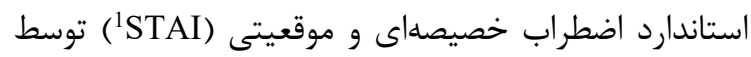
يزوهشكر در بخش يست آنزيو براى بيماران خوانده و وراسخ

$$
\text { سؤالات ثبت شد. }
$$

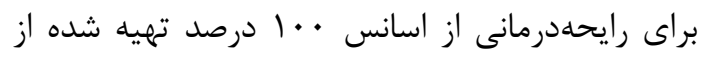

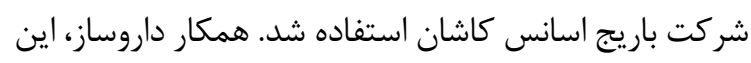

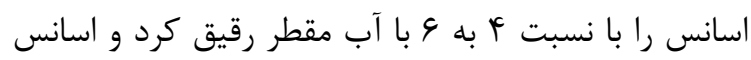

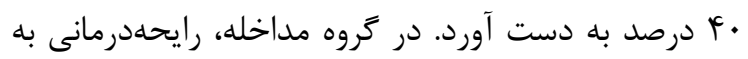

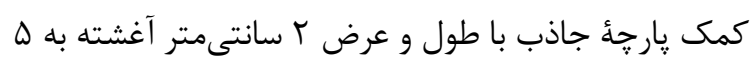

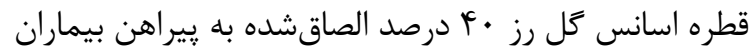

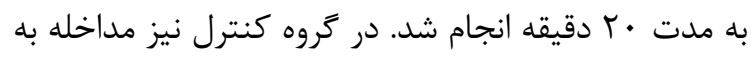

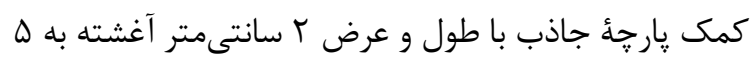

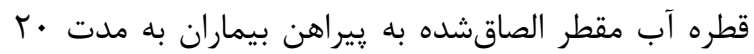

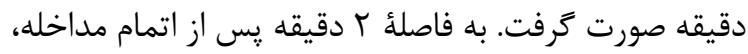

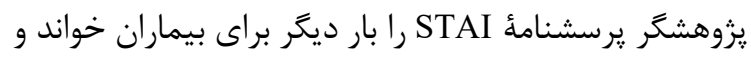

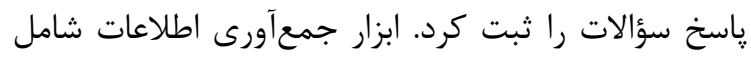

ترس، درد، ناراحتى، محدوديت حركت و اضطراب همراه

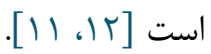

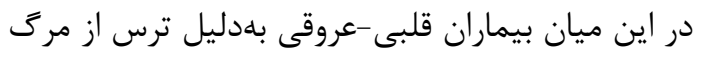

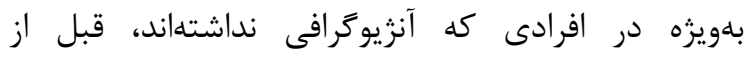
آنزيوكرافى عروق كرونرى سطوحى از اضطراب را تحمل

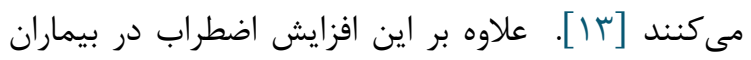

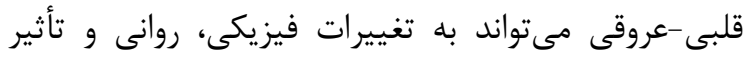

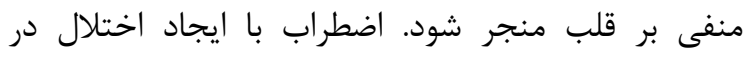

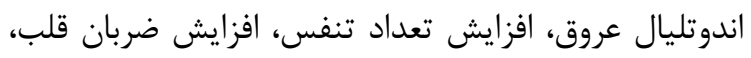

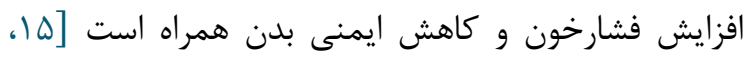

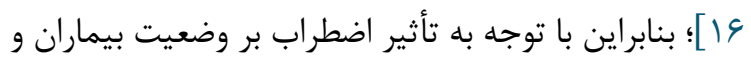

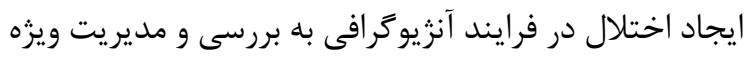
نياز است [19]. درمانهاى مكمل از جمله ماساز درمانى، طب سوزنى و

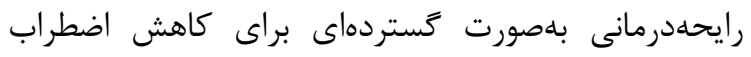
بيماران استفاده مىشود [IV]

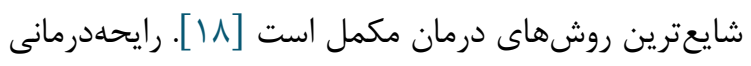
به استفاده از اسانس كياهان معطر براى مصارف طبى كفتهان

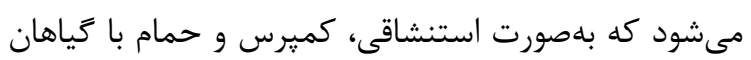
و مواد معدنى صورت مى گيرد و در كاهش درد، كمئ استرس،

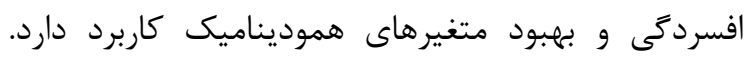

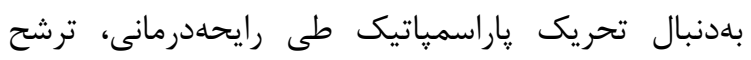

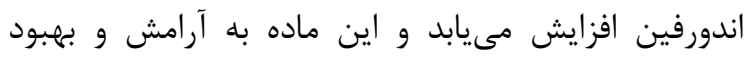

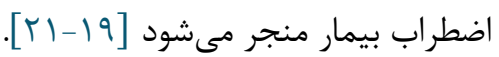

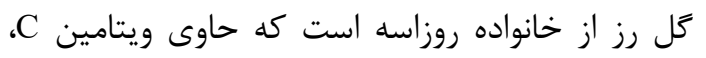

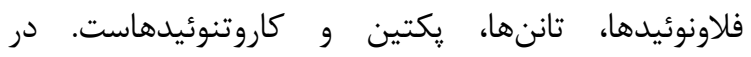

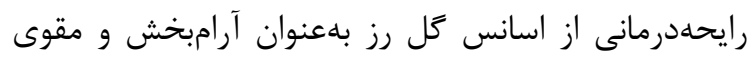

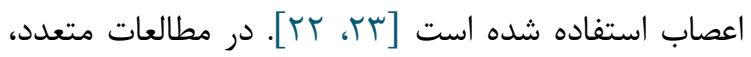
تأثيرات اسانس گل رز با اثر سايرسيو بر سيستم سمياتيك و

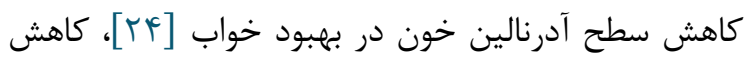

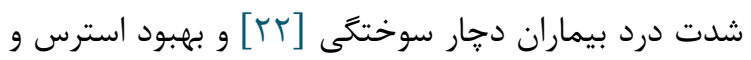

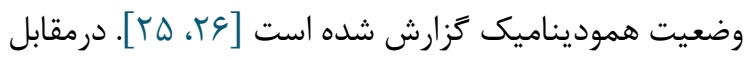
مطالعات ديخر نشاندهنده تأثيرنداشتن رايحهدر مانى هستند

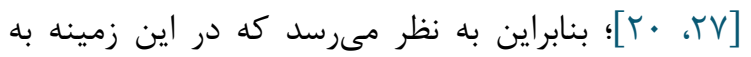

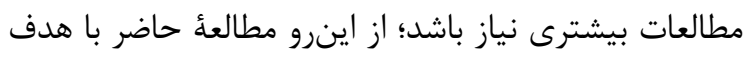

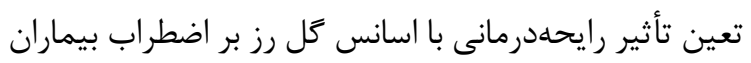

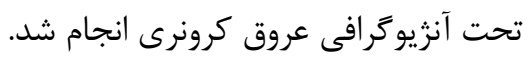

\footnotetext{
1. State-Trait Anxiety Inventory
}

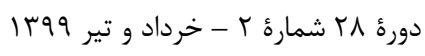


9 تأثير رايحهدرمانى استنشاقى با اسانس كل رز بر اضطراب بيماران..

كرونباخ = r/9 •)، آزمون مجدد (r=/9V) و روايى نسخd

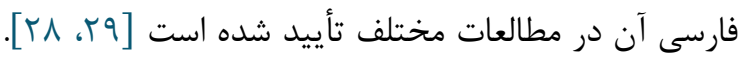

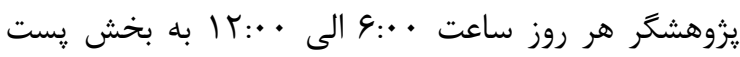

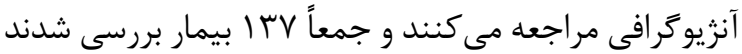
كه • 11 بيمار معيارهاى ورود به مطالعه را داشتند و بركأ رضايتنامه ورود به مطالعه را امضا كردند. طى مطالعه در

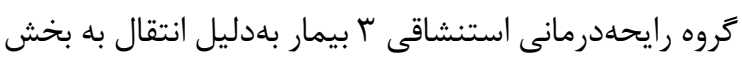
آنزيوكرافى و ץ بيمار بهعلت تحمل نكردن رايحه از مطالعه

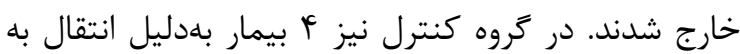

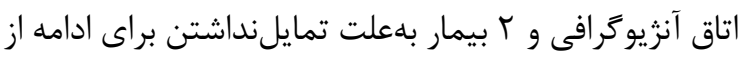

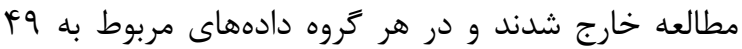

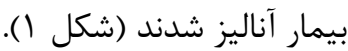

• 1 سؤال جمعيت شناختى (جنس، وضعيت تأهل، سن، سطح سواد، محل سكونت، سابقه بسترى، مصرف سيخار،

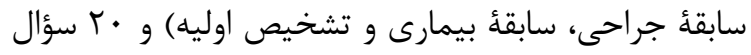
اضطراب موقعيتى STAI بود. در اين مطالعه، بخش اضطراب موقعيتى يرسشنامه

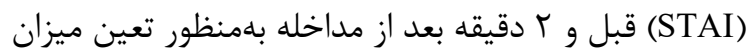

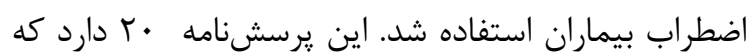

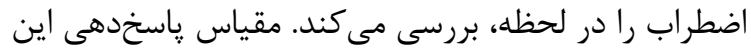

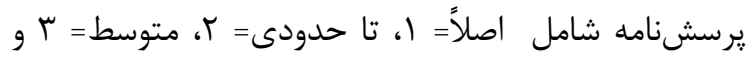

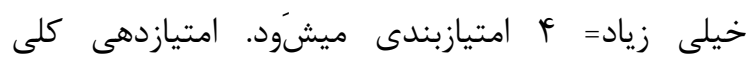

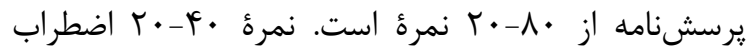

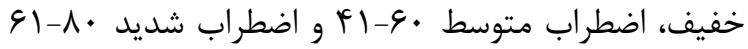

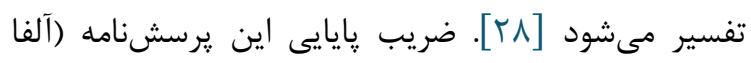

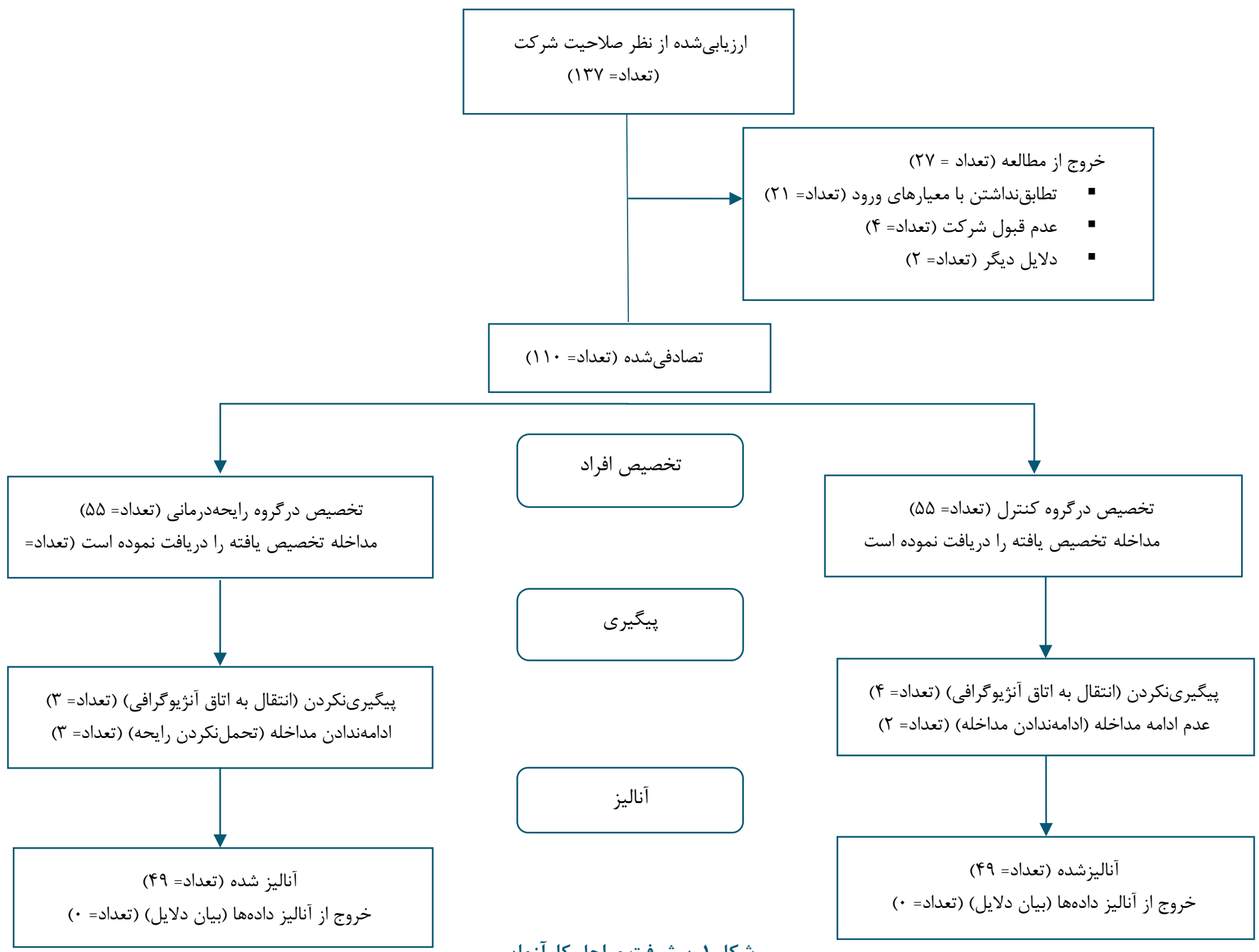


مطلع نبود. براى اين منظور يزوهشكر طى مداخله از ماسك إسك

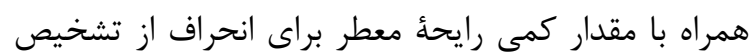

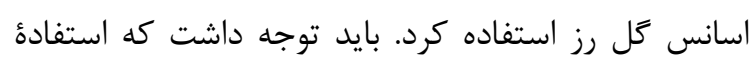

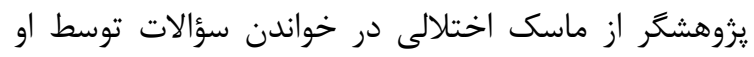

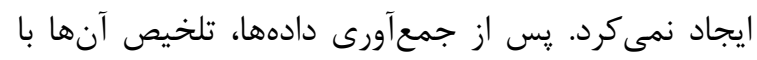

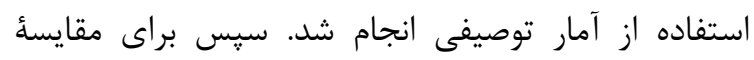

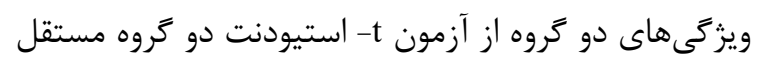

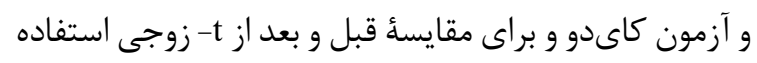

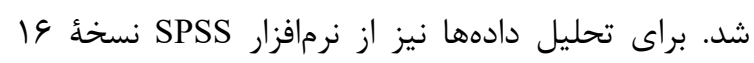
(SPSS Inc., Chicago, Ill., USA) معنى دارى آزمونها نيز هـ درصد در نظر كرفته شد.

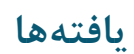

ميانكَين سن بيماران تحت آنزيوكرافى در كروه رايحهدرمانى

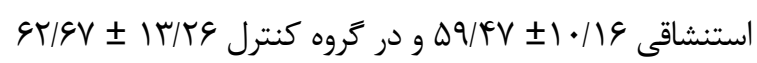

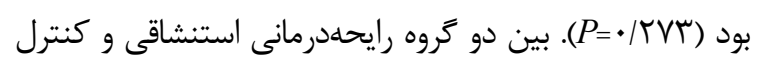

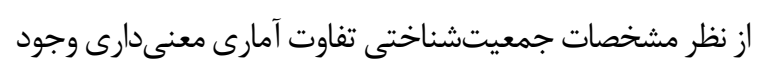

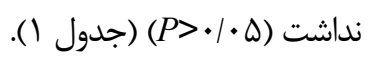

براى تخصيص تصادفى بيماران به دو كروه رايحهدرمانى

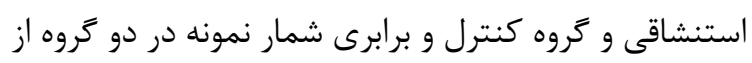

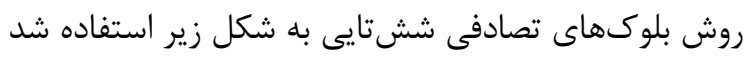

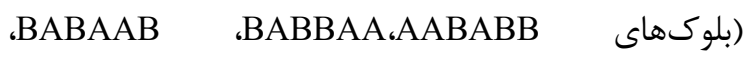
‘BBAABA ‘BAAABB ،AABBAB ،ABAABB

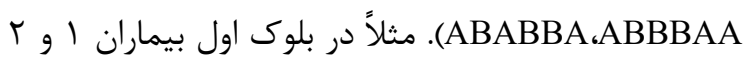

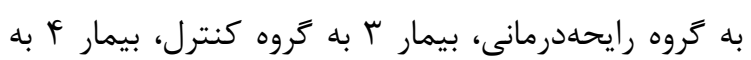

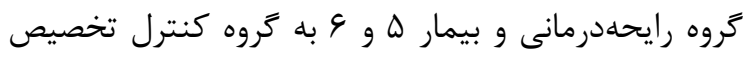

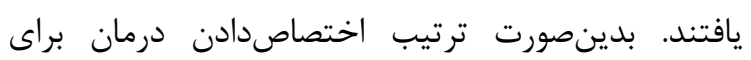

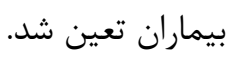
اختصاص گروه رايحهدرمانى استنشاقى و گروه كنترل به حروف A و B بهصورت تصادفى را دستيار يزوهشكر و و بلوكبندى و اجراى مطالعه را يزوهشكر انجام دادند. در اين

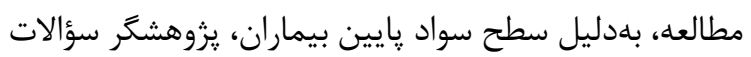

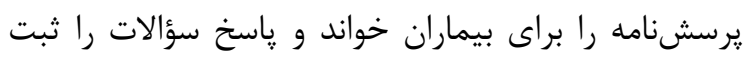

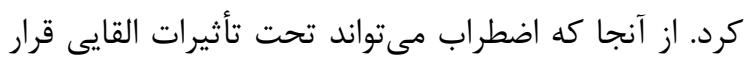

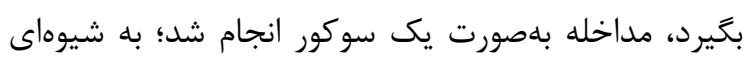

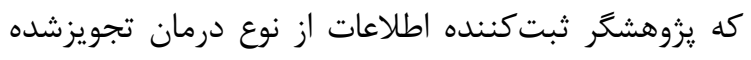

جدول ا. مشخصات جمعيت شناختى بيماران تحت آنزيوكرافى عروق كرونرى در گروه رايحهدرمانى و كروه كنترل

\begin{tabular}{|c|c|c|c|c|}
\hline \multirow{2}{*}{ معنى مارى } & كروه كنترل (Fq) & تروه رايحهدر مانى & \multicolumn{2}{|c|}{ Groups } \\
\hline & تعداد (درصد) & تعداد (درصد) & \multicolumn{2}{|c|}{ مشخصات جمعيتشناختى } \\
\hline \multirow{2}{*}{$\cdot \mid 8 \wedge 9$} & (DI) rQ & $r(\varphi \in / 9)$ & مرد مرد & \multirow{2}{*}{ جنس } \\
\hline & $(F q) Y^{q}$ & $r \varepsilon(\Delta r / l)$ & 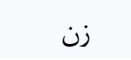 & \\
\hline \multirow{3}{*}{$\cdot / T F V$} & $(\Lambda) / 9) F$. & $\operatorname{Rf}(9 / / \wedge)$ & متأهل & \multirow{3}{*}{ وضعيت تأهل } \\
\hline & ( $(r) 1$ & $r(r / 1)$ & مجرد & \\
\hline & $(\mid \varepsilon / \Gamma) \wedge$ & $r(9 / 1)$ & بيوه & \\
\hline \multirow{5}{*}{.$/ T V R$} & $\Delta(1 \cdot / r)$ & $(9 / 1) r$ & $r \Delta-F q$ & \multirow{5}{*}{ سن (سال) } \\
\hline & $(\mid F / T) V$ & $(Y T / Y) \backslash 1$ & $F \Delta-\Delta F$ & \\
\hline & $(Y T / V) 19$ & זr (Fe/9) & $\Delta \Delta-s q$ & \\
\hline & (Y\&|Q) & $(\mid f / T) V$ & $\varphi \Delta-V \Delta$ & \\
\hline & $(\mid \varepsilon / \Gamma) \wedge$ & $(1 \cdot / r) \Delta$ & $<\mathrm{V} Q$ & \\
\hline \multirow{4}{*}{.$/ 1 \vee 9$} & $(V / / \Delta) r_{G}$ & $(\Delta q / r) r q$ & 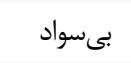 & \multirow{4}{*}{ سطح سواد } \\
\hline & $(Y F / Q) I T$ & $(T N / 9) \mid F$ & 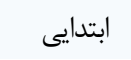 & \\
\hline & $(\cdot) \cdot$ & $(9 / 1) r$ & راهنمايى & \\
\hline & (Y) 1 & $(g / 1) r$ & دانشعاهى & \\
\hline \multirow{2}{*}{$\cdot / r \cdot \Lambda$} & (DI) TD & $(\Phi) / T) \Gamma \cdot$ & شهر & \multirow{2}{*}{ محل سكونت } \\
\hline & $(q q) T F$ & $($ MN/N) 19 & 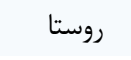 & \\
\hline \multirow{2}{*}{.$/ 99 V$} & r & $(Y Y / Y) 11$ & . & \multirow{2}{*}{ سابقه بسترى } \\
\hline & $(\Delta \Delta / 1) T V$ & $(\Delta \Delta / 1) T V$ & 1-r & \\
\hline
\end{tabular}


91 تأثير رايحهدرمانى استنشاقى با اسانس كل رز بر اضطراب بيماران..

\begin{tabular}{|c|c|c|c|c|}
\hline \multirow{2}{*}{ معنى دارى } & كروه كنترل (Fq) & تروه رايحهدرمانى & \multicolumn{2}{|l|}{ Groups } \\
\hline & تعداد (درصد) & تعداد (درصد) & مشخصات جمعيتشناختى & \\
\hline \multirow{2}{*}{.$/ 998$} & $(\mid f / \mu) V$ & $\left(\mid N / F^{\mathcal{F}}\right) q$ & $4-9$ & \multirow{2}{*}{ سابقه بسترى } \\
\hline & $(F / 1) r$ & $(F / 1) r$ & $<\varphi$ & \\
\hline \multirow{2}{*}{$\cdot / T 19$} & $(T F / Q) \mid T$ & $(\mid \varepsilon / \Gamma) \wedge$ & بله & \multirow{2}{*}{ مصرف سيخار } \\
\hline & $(V \Delta / \Delta) \Gamma V$ & $(\Lambda \Gamma / V) \uparrow l$ & خير & \\
\hline \multirow{2}{*}{ - / $\Delta \&$} & r & $(\varepsilon / / r) r$. & بله & \multirow{2}{*}{ سابقهُ جراحى } \\
\hline & 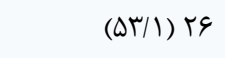 & $(ז / \Lambda) 19$ & 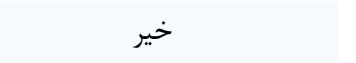 & \\
\hline \multirow{7}{*}{$.19 \cdot 1$} & $(\Delta \Delta / I) T V$ & $(F q) T F$ & بدون سابقه & \multirow{7}{*}{ سابقع بيمارى } \\
\hline & $(N / T) F$ & $(\mid F / r) V$ & ديابت شيرين & \\
\hline & $(9 / 1) r$ & $(\mathcal{N} / Y)^{\uparrow}$ & بيمارى توارشى & \\
\hline & $(\mathbb{F} / 1) T$ & $(\mathbb{F} / 1) T$ & 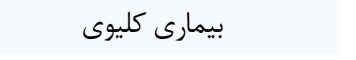 & \\
\hline & $(\mid \varepsilon / \Gamma) \wedge$ & $\left(\mid N / T^{\mathbb{4}}\right)^{9}$ & بيمارى عروق كرونرى & \\
\hline & $(9 / 1) r$ & $(r) 1$ & بيمارى نورولوزيك & \\
\hline & $(\boldsymbol{F} / 1) r$ & $(r / 1) r$ & ديخر موارد & \\
\hline \multirow{5}{*}{. } & $(Y \cdot / \varphi))$ & $(T Y / F) \mid r$ & درد قفسأه سينه & \multirow{5}{*}{ تشخيص اوليه } \\
\hline & (rg/Q) & $(T r / V) 19$ & تنغَى نفس & \\
\hline & $(Y K / V) I V$ & (YG|Q) IT & ت ت تنكى عروق & \\
\hline & $(\mid \mathcal{F} / T) \vee$ & $(19 / \Gamma) \wedge$ & درد قفسئ سينه و تنكَىنفس & \\
\hline & $(F / 1) r$ & $(r) 1$ & ديخر موارد & \\
\hline
\end{tabular}

مقايسة ميانگين اضطراب دو گروه رايحهدرمانى استنشاقى

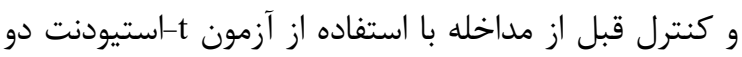

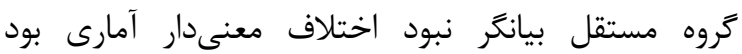

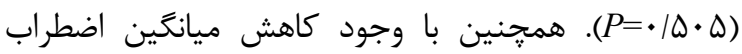

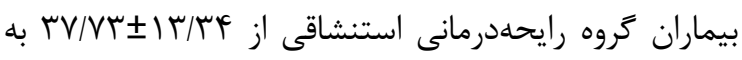

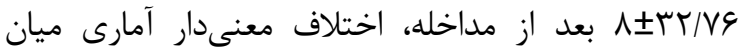

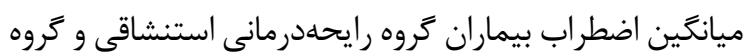

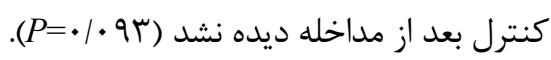

در ₹روه رايحهدرمانى قبل از مداخله VI/Y درصد

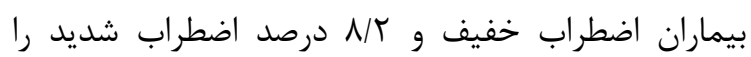

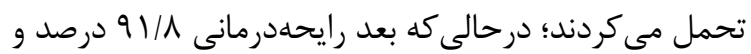

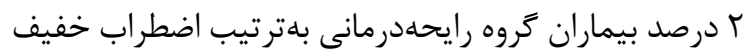

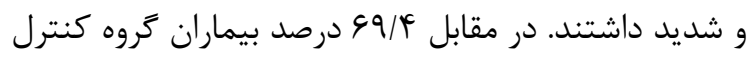
اضطراب خفيف داشتند و / / أ درصد اضطراب شديد داشتند.

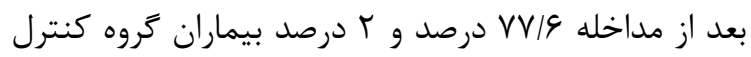
بلهترتيب اضطراب خفيف و شديد داشتند (جدول مداحل).

جدول ז. سطح اضطراب بيماران تحت آنثيوگر افى عروق كرونرى در گروه رايحهدرمانى و َروه كنترل

\section{بعد از مداخله}

\section{قبل از مداخله}

\section{نوبت}

\begin{tabular}{|c|c|c|c|c|}
\hline كنترل & رايحهدرمانى & كنترل & رايحهدرمانى & كروه \\
\hline تعداد (درصد) & تعداد (درصد) & تعداد (درصد) & تعداد (درصد) & اضطراب \\
\hline 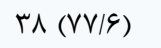 & $f Q(9) / \Lambda)$ & $\mu \mathcal{F}(\xi q / \mathcal{F})$ & $r \Delta(V / / \mathcal{C})$ & خفيف \\
\hline $1 \cdot(Y \cdot / \mathbb{Y})$ & $r(\xi / 1)$ & $\Pi(Y \& \mid \Delta)$ & $1 \cdot(Y \cdot / \mathbb{F})$ & متوسط \\
\hline $1(T)$ & $1(r)$ & $r(r / 1)$ & $F(\Lambda / r)$ & شديد \\
\hline$q q(1 \cdots)$ & $p q(1 \cdots)$ & $p q(1 \cdots)$ & $p q(1 \cdots)$ & مجموع \\
\hline
\end{tabular}

زوجى بيانگر وجود اختلاف معنى دار آمارى بين اضطراب قبل و

ميانگين اضطراب بيماران در گروه رايحهدرمانى استنشاقى

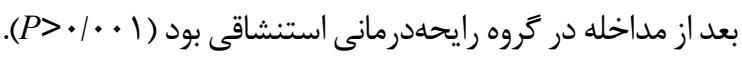

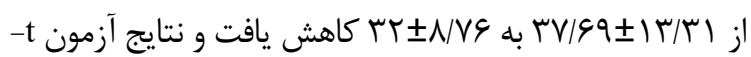


زوجى بيانكر نبود تفاوت معنىدار آمارى بين قبل و بعد از

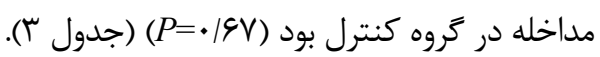

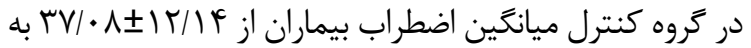

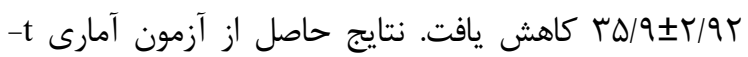

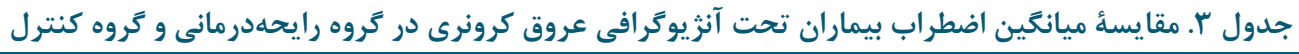

\begin{tabular}{|c|c|c|c|c|c|c|}
\hline معنى & t & خطاى & ت ت تفاوت & تروه كنترل & تروه رايحهدرمانى & \multirow{2}{*}{ اضطراب } \\
\hline دارى & 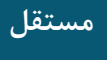 & استاندارد & ميانگينها & ميانگين 土 انحراف معيار & ميانكَين 土 انحراف معيار & \\
\hline $14 q 4$ & •/rar & $r / \Delta \Lambda$ & . $19 \Delta r$ & $r V / \cdot \Lambda \pm|r /|^{f}$ & $r V / V T \pm I r / T r$ & اضطراب قبل از مداخله \\
\hline \multirow[t]{4}{*}{1.91} & $-1 / 9 V \mu$ & $1 / 19$ & س & $r \Delta / r \pm 9 / 9 r$ & $r T \pm \Lambda / V G$ & اضطراب بعد از مداخله \\
\hline & & & & מיזיו & אוTו & خطاى استاندارد \\
\hline & & & & I/AVQ & אس & آمارة t زوجى \\
\hline & & & & $.1 .9 \mathrm{~V}$ & $<\cdot / \cdot \cdot 1$ & معنى دارى \\
\hline
\end{tabular}

قاعدگى مؤثر است [بس]] كه اين نتايج با نتايج مطالعه حاضر همسو بود. در مطالعهاى ديخر، Dagli و همكاران به بررسى إنى

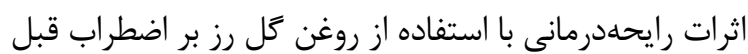

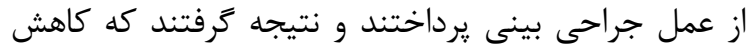

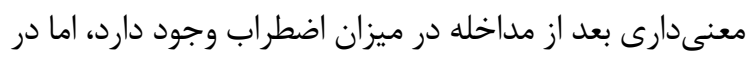

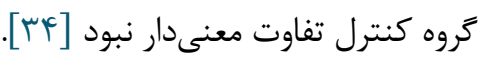
در مقابل نتايج مطالعه Babaii و همكاران نشاندهندة

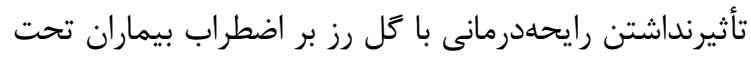

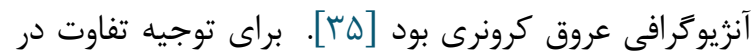

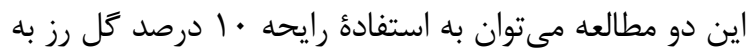

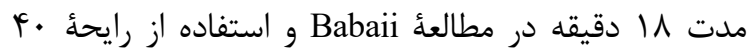

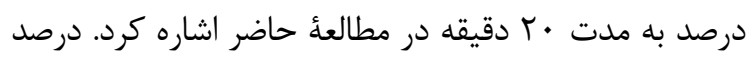
رايحٔٔ مورد استفاده و مدتزمان رايحهدرمانى بلهصورت

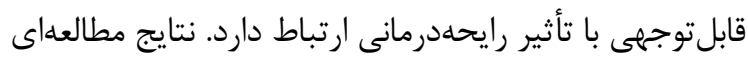

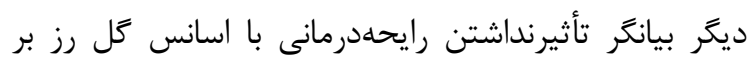

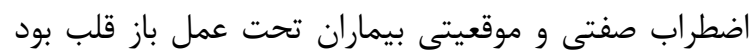

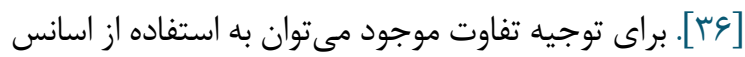

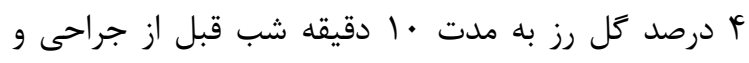
بلافاصله قبل از جراحى جامعه در مطالعه ذكرشده اشاره كرد. با وجود بيشتربودن دفعات رايحهدرمانى، درصد اسانس

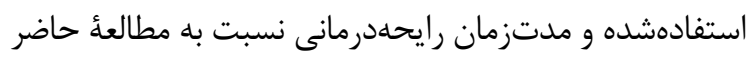

محدوديتهاى مطالعه حاضر به حجم نمونهُ كم آن مربوط

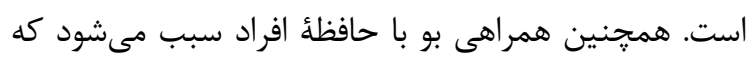

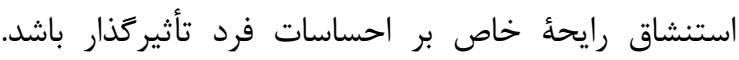
همجنين استنشاق رايحهاى همراه با خاطرات ناخوشايند

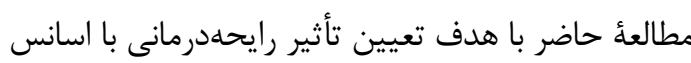

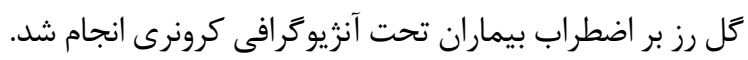
نتايج اين مطالعه نشان مىدهد استفاده از رايحهدرمانى با بان

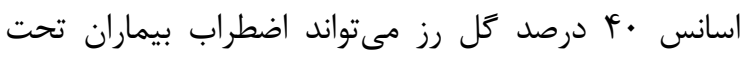

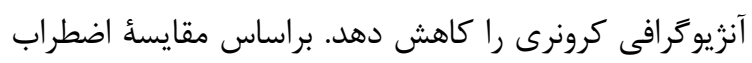
نيز اضطراب كروه رايحهدرمانى برخلاف كروه كنترل كاهش

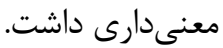
Neisani ، Kheirkhah

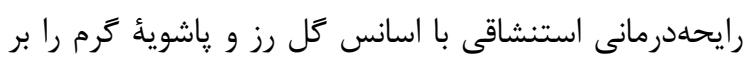
اضطراب زنان نخستزا طى فاز اول زايمان بررسى كردند و وانس

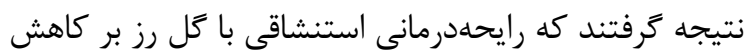

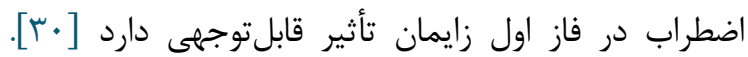
همجنين نتايج مطالعُ مشابه Hamdamian و همكاران بيانكر

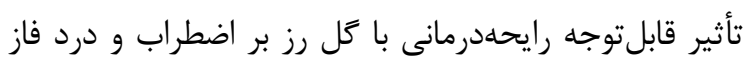

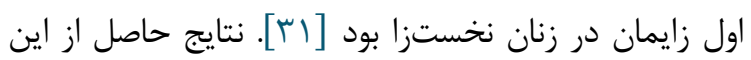
مطالعات با نتايج مطالعهُ حاضر همخوانى دارد. Karadag و همكاران مطالعهاى را با هدف بررسى تأثير رايحهدرمانى با بارئ

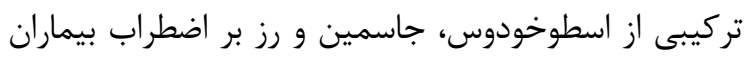
بسترى در بخش ويزه انجام دادند و نتيجه كرفتيند كه كه رايحهدرمانى بر كاهش اضطراب بيماران تأثيركذار است كند

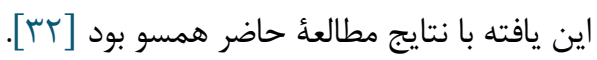

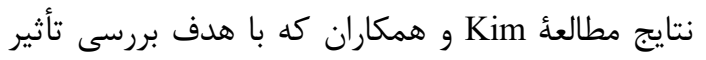
رايحلدرمانى بلصورت ماساز شكم با تركيبى از اسانس كل رز بر درد و اضطراب ناشى از قاعدىى در يرستاران انجام شد،

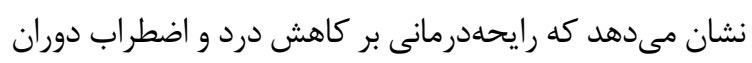




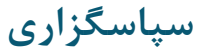

اين مقاله قسمتى از نتايج پايانامأهُ كارشناسى ارشد

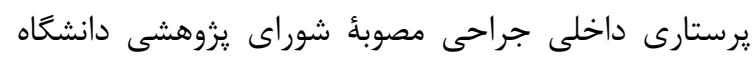

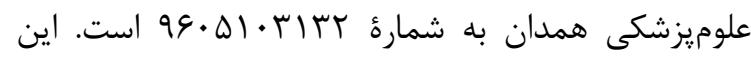
طرح در كميتهٔ اخلاق با كد

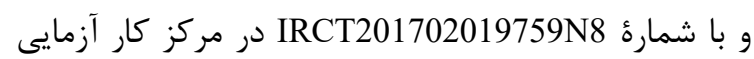

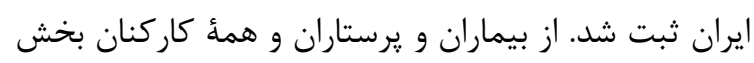

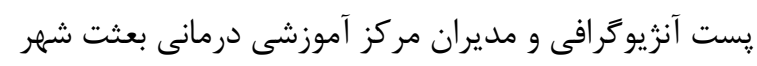
همدان تشكر و قدردانى مى شود.

$$
\text { تعارض در منافع }
$$

بين نويسندًان هيجَّونه تعارضى در منافع وجود ندارد

$$
\text { منابع مالى من مالى }
$$

منابع مالى اين مطالعه توسط نويسندكان تامين شده است.

\section{References}

1. Reamy BV, Williams PM, Kuckel DP. Prevention of cardiovascular disease. Primary Care: Clinics in Office Practice. $2018 \quad$ Mar 1;45(1):25-44. [DOI:10.1016/i.pop.2017.11.003] [PMID]

2. Sacks FM, Lichtenstein $\mathrm{AH}, \mathrm{Wu} \mathrm{JH}$, Appel LJ, Creager MA, Kris-Etherton PM, Miller M, Rimm EB, Rudel LL, Robinson JG, Stone NJ. Dietary fats and cardiovascular disease: a presidential advisory from the American Heart Association. Circulation. $2017 \mathrm{Jul}$ 18;136(3):e1-23. [DOI:10.1161/CIR.0000000000000510] [PMID]

3. Yaghoobzadeh A, Soleimani MA, Allen KA, Chan YH, Herth KA. Relationship between spiritual wellbeing and hope in patients with cardiovascular disease. Journal of religion and health. 2018 Jun 1;57(3):93850. [DOI:10.1007/s10943-017-0467-0] [PMID]

4. Fernández-Rodríguez D, Grillo-Pérez JJ, PérezHernández H, Rodríguez-Esteban $\mathrm{M}$, Pimienta $\mathrm{R}$, Acosta-Materán C, Rodríguez S, Yanes-Bowden G, Vargas-Torres MJ, Flecha AS, Hernández-Afonso J. Evaluación prospectiva del desarrollo de nefropatía inducida por contraste en pacientes con síndrome coronario agudo tratados con angiografía coronaria rotacional vs. angiografía coronaria convencional: Estudio CINERAMA. nefrologia. 2018 Mar 1;38(2):169-78. [DOI:10.1016/j.nefro.2017.05.011] [PMID]

5. The SC. CT coronary angiography in patients with suspected angina due to coronary heart disease (SCOT-HEART): an open-label, parallel-group, multicentre trial. The Lancet. 2015 Jun

$$
\begin{aligned}
& \text { سبب پيامدهايى منفى از جمله افزايش اضطراب مىشود. براى }
\end{aligned}
$$

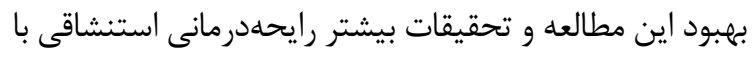

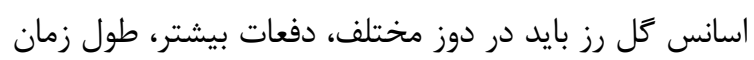

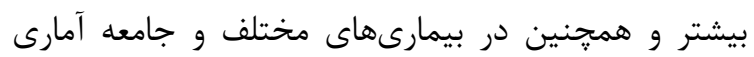

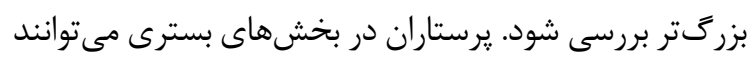

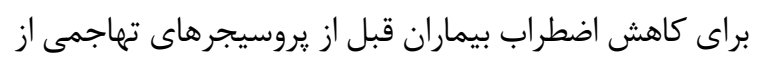

$$
\begin{aligned}
& \text { رايحلدرمانى با كل رز استفاده كنند. } \\
& \text { نتيجه گيرى } \\
& \text { رايحهدرمانى با اسانس • F درصد ₹ل رز اضطراب بيماران }
\end{aligned}
$$

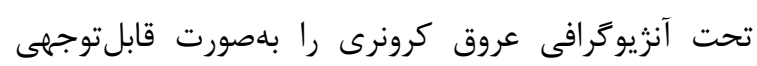

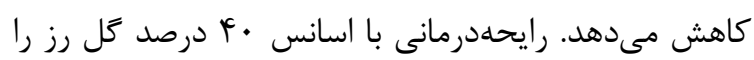

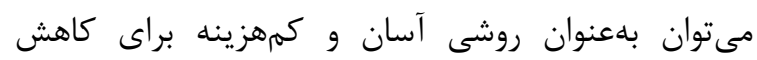

$$
\begin{aligned}
& \text { اضطراب بيماران قبل از آنزيوكرافى عروق كرونرى در كنار } \\
& \text { درمانهاى دارويى استفاده كرد. }
\end{aligned}
$$

13;385(9985):2383-91. 6736(15)60291-4]

[DOI:10.1016/S0140-

6. Eckert J, Schmidt M, Magedanz A, Voigtländer T, Schmermund A. Coronary CT angiography in managing atherosclerosis. International journal of molecular sciences. 2015 Feb;16(2):3740-56. [DOI:10.3390/ijms 16023740] [PMID] [PMCID]

7. Mobini-Bidgoli M, Taghadosi M, Gilasi H, Farokhian A. The effect of hand reflexology on anxiety in patients undergoing coronary angiography: A singleblind randomized controlled trial. Complementary therapies in clinical practice. 2017 May 1;27:31-6. [DOI:10.1016/j.ctcp.2017.01.002] [PMID]

8. Arami S, Kazemi M, Esmaeili-nadimi A. Comparing the effect of acupressure points shenmen (HE7) with a third eye on anxiety in patients undergoing coronary angiography. Medical-Surgical Nursing Journal. 2015;4(2):41-6.

9. Foji S, Tadayonfar MA, Mohsenpour M, Rakhshani MH. The study of the effect of guided imagery on pain, anxiety and some other hemodynamic factors in patients undergoing coronary angiography. Complement Ther Clin Pract. 2015;21(2):119-23. [DOI:10.1016/j.ctcp.2015.02.001] [PMID]

10. de Agustin JA, Marcos-Alberca P, Fernández-Golfin C, Feltes G, Nuñez-Gil IJ, Almeria C, Rodrigo JL, Arrazola J, de Isla LP, Macaya C, Zamorano J. Should computed tomography coronary angiography be aborted when the calcium score exceeds a certain threshold in patients with chest pain?. International journal of cardiology. 2013 Sep 1;167(5). [DOI:10.1016/j.ijcard.2012.05.041] [PMID] 
11. Dogan MV, Senturan L. The effect of music therapy on the level of anxiety in the patients undergoing coronary angiography. Open J Nurs. 2012;2(03):165. [DOI:10.4236/ojn.2012.23025]

12. Lukies MW, Richmond D, Hornsey EK, Paterson G, Ko PH, Chuen J, Ranatunga D, Edelman RR, Lim RP. Patient Perception of Lower Limb Non-Contrast Magnetic Resonance Angiography and Digital Subtraction Angiography in Diabetic Patients with Peripheral Arterial Disease. Cardiovascular Imaging Asia. $2017 \quad$ Oct $1 ; 1(4): 240-7$. [DOI:10.22468/cvia.2017.00087]

13. Gökçe E, Arslan S. Possible effect of video and written education on anxiety of patients undergoing coronary angiography. Journal of PeriAnesthesia Nursing. 2019 Apr [DOI:10.1016/j.jopan.2018.06.100] [PMID]

14. Hur MH, Song JA, Lee J, Lee MS. Aromatherapy for stress reduction in healthy adults: a systematic review and meta-analysis of randomized clinical trials. Maturitas. $2014 \quad$ Dec 1;79(4):362-9. [DOI:10.1016/j.maturitas.2014.08.006] [PMID]

15. Delewi R, Vlastra W, Rohling WJ, Wagenaar TC, Zwemstra M, Meesterman MG, Vis MM, Wykrzykowska JJ, Koch KT, de Winter RJ, Baan Jr J. Anxiety levels of patients undergoing coronary procedures in the catheterization laboratory. International Journal of Cardiology. 2017 Feb 1;228:926-30. [DOI:10.1016/j.ijcard.2016.11.043] [PMID]

16. Kalogianni A, Almpani P, Vastardis L, Baltopoulos G, Charitos C, Brokalaki H. Can nurse-led preoperative education reduce anxiety and postoperative complications of patients undergoing cardiac surgery? Eur J Cardiovasc Nurs. 2016;15(6):447-58. [DOI:10.1177/1474515115602678] [PMID]

17. Çetinkaya F, Aşiret GD, Yilmaz CK, İnci S. Effect of listening to music on anxiety and physiological parameters during coronary angiography: A randomized clinical trial. European Journal of Integrative Medicine. 2018 Oct 1;23:37-42. [DOI:10.1016/j.eujim.2018.09.004]

18. Song JA, Lee MK, Min E, Kim ME, Fike G, Hur MH. Effects of aromatherapy on dysmenorrhea: A systematic review and meta-analysis. International journal of nursing studies. 2018 Aug 1;84:1-1. [DOI:10.1016/j.ijnurstu.2018.01.016] [PMID]

19. Steflitsch W, Steflitsch M. Clinical aromatherapy. Journal of Men's Health. 2008 Mar;5(1):74-85. [DOI:10.1016/j.jomh.2007.11.001]

20. Bikmoradi A, Seifi Z, Poorolajal J, Araghchian M, Safiaryan R, Oshvandi K. Effect of inhalation aromatherapy with lavender essential oil on stress and vital signs in patients undergoing coronary artery bypass surgery: A single-blinded randomized clinical trial. Complementary therapies in medicine. 2015 Jun 1;23(3):331-8. [DOI:10.1016/i.ctim.2014.12.001] [PMID]

21. Johnson JR, Rivard RL, Griffin KH, Kolste AK, Joswiak D, Kinney ME, Dusek JA. The effectiveness of nurse-delivered aromatherapy in an acute care setting. Complementary therapies in medicine. 2016 Apr 1;25:164-9. [DOI:10.1016/j.ctim.2016.03.006] [PMID]

22. Bikmoradi A, Harorani M, Roshanaei G, Moradkhani $\mathrm{S}$, Falahinia GH. The effect of inhalation aromatherapy with damask rose (Rosa damascena) essence on the pain intensity after dressing in patients with burns: A clinical randomized trial. Iranian journal of nursing and midwifery research. 2016 May;21(3):247. [DOI:10.4103/1735-9066.180380] [PMID] [PMCID]

23. Boskabady MH, Shafei MN, Saberi Z, Amini S. Pharmacological effects of Rosa damascena. Iranian journal of basic medical sciences. $2011 \mathrm{Jul} ; 14(4): 295$.

24. Hajibagheri A, Babaii A, Adib-Hajbaghery M. Effect of Rosa damascene aromatherapy on sleep quality in cardiac patients: a randomized controlled trial. Complementary therapies in clinical practice. 2014 Aug [DOI:10.1016/j.ctcp.2014.05.001] [PMID]

25. Nategh M, Heidari MR, Ebadi A, Kazemnejad A, Babaei Beigi MA. Effect of lavender aromatherapy on hemodynamic indices among patients with acute coronary syndrome: a randomized clinical trial. Iran J Crit Care Nurs. 2015 Jan 1;7(4):201-8.

26. Lee MK, Lim S, Song JA, Kim ME, Hur MH. The effects of aromatherapy essential oil inhalation on stress, sleep quality and immunity in healthy adults: Randomized controlled trial. European Journal of Integrative Medicine. 2017 Jun 1;12:79-86. [DOI:10.1016/j.eujim.2017.04.009]

27. Abdelhakim, Ahmed Mohamed, et al. "The effect of inhalation aromatherapy in patients undergoing cardiac surgery: A systematic review and metaanalysis of randomized controlled trials." Complementary Therapies in Medicine 48 (2020): 102256. [DOI:10.1016/j.ctim.2019.102256] [PMID]

28. Mayou RA, Gill D, Thompson DR, Day A, Hicks N, Volmink J, Neil A. Depression and anxiety as predictors of outcome after myocardial infarction. Psychosomatic medicine. 2000 Mar 1;62(2):212-9. [DOI:10.1097/00006842-200003000-00011] [PMID]

29. Kalkhoran MA, Karimollahi M. Religiousness and preoperative anxiety: a correlational study. Annals of General Psychiatry. 2007 Dec;6(1):17. [DOI:10.1186/1744-859X-6-17] [PMID] [PMCID]

30. Kheirkhah M, Neisani L, Haghani H. Comparison of the effects of aromatherapy with essential oils of damask rose and hot footbath on the first stage of labor anxiety in Nulliparous women. Complementary Medicine Journal of faculty of Nursing \& Midwifery. 2012;2(2):126-34. (persian).

31. Hamdamian S, Nazarpour S, Simbar M, Hajian S, Mojab F, Talebi A. Effects of aromatherapy with Rosa damascena on nulliparous women's pain and anxiety of labor during first stage of labor. Journal of integrative medicine. $2018 \quad$ Mar 1;16(2):120-5. [DOI:10.1016/j.joim.2018.02.005] [PMID]

32. Karadag E, Samancioglu S, Ozden D, Bakir E. Effects of aromatherapy on sleep quality and anxiety of

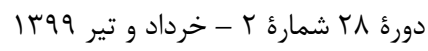


patients. Nursing in critical care. 2017 Mar;22(2):10512. [DOI:10.1111/nicc.12198] [PMID]

33. Kim YJ, Lee MS, Yang YS, Hur MH. Selfaromatherapy massage of the abdomen for the reduction of menstrual pain and anxiety during menstruation in nurses: a placebo-controlled clinical trial. European journal of integrative medicine. 2011 Sep 1;3(3):e165-8. [DOI:10.1016/j.eujim.2011.08.007]

34. Dagli R, Avcu M, Metin M, Kiymaz S, Ciftci H. The effects of aromatherapy using rose oil (Rosa damascena Mill.) on preoperative anxiety: A prospective randomized clinical trial. European Journal of Integrative Medicine. 2019 Feb 1;26:37-42. [DOI:10.1016/j.eujim.2019.01.006]

35. Babaii A, Abbasinia M, Hejazi SF, Seyyed Tabaei SR, Dehghani F. The effect of listening to the voice of Quran on anxiety before cardiac catheterization: a randomized controlled trial. Health, Spirituality and Medical Ethics. 2015 Jun 10;2(2):2-7.

36. Fazlollahpour-Rokni F, Shorofi SA, Mousavinasab N, Ghafari R, Esmaeili R. The effect of inhalation aromatherapy with rose essential oil on the anxiety of patients undergoing coronary artery bypass graft surgery. Complementary therapies in clinical practice. 2019 Feb 1;34:201-7. [DOI:10.1016/j.ctcp.2018.11.014] [PMID] 